\title{
Alterações retinianas em jovens portadores de anemia falciforme (hemoglobinopatias) em hospital universitário no nordeste do Brasil
}

\author{
Retinal impairment in young individuals with sickle cell anemia (hemoglobin SS disease) \\ in university hospital in Northeastern of Brazil
}

Allisson Mário dos Santosin ${ }^{1}$, Gustavo Baptista de Almeida Faro², Marcus Vinicius Melo do Amaral ${ }^{3}$, Cristiano de Queiroz Mendonça ${ }^{4}$, Bruno Campelo Leal ${ }^{4}$, Rosana Cipolotti ${ }^{3}$

\section{RESUMO}

Objetivos: Descrever e classificar alterações retinianas encontradas em portadores de anemia falciforme com genótipo SS, bem com comparar métodos diagnósticos (mapeamento de retina e angiofluoresceinografia).

Métodos: Neste estudo transversal foram avaliados pacientes portadores de anemia falciforme com idade igual ou superior a sete anos. Esses pacientes foram submetidos a mapeamento de retina e angiofluoresceinografia. Os achados do mapeamento de retina foram agrupados em três classes: sem alterações; alterações não proliferativas e alteraçôes proliferativas. Os resultados à angiofluoresceinografia foram classificados de acordo com os estágios de Goldberg, variando de I a V e expressando gradiente crescente de gravidade.

Resultados: Foram avaliados 61 olhos de 31 pacientes. A retinopatia falciforme foi encontrada em 38/61 (62,3\%) dos olhos examinados. A média de idade do grupo de portadores de retinopatia foi menor que dos pacientes sem retinopatia ( 14,4 versus $17,4$ anos, $p=0,04)$. Observou-se elevada freqüência de retinopatia não proliferativa, especialmente as tortuosidades vasculares $(27,9 \%)$, seguidas por anastomoses arteriovenosas na periferia da retina (24,6\%) e oclusões arteriolares (8,2\%). Em um olho foi observado neovascularização. Em 16,4\% dos olhos obteve-se resultado normal no mapeamento de retina e alterado à angiofluoresceinografia.

Conclusões: As alterações retinianas do tipo não proliferativa são frequentes e precoces nos portadores de anemia falciforme do tipo SS, sendo a angiofluoresceinografia mais sensível no diagnóstico quando comparada ao mapeamento de retina.

Descritores: Anemiafalciforme; Retina/fisiopatologia;Doenças retinianas/diagnóstico; Angiofluoresceinografia/metodos; Oftalmoscopia

\begin{abstract}
Purposes: To describe and categorize retinal vascular changes in patients with sickle cell anemia, as well as to compare diagnostic methods (indirect ophthalmoscopy and fluorescein angiography).

Methods: Patients with sickle cell anemia over the age of seven were examined. Complete ophthalmologic examination with indirect ophthalmoscopy and angiography was performed in each patient. The fundoscopy results were grouped in 3 classes: normal; non- proliferative retinopathy, which includes vasculartortuosity, blacksunburst, salmon-patch and peripheral closure/anastomoses; and proliferative retinopathy, related to neovascular proliferation. Angiography results were classified according to Goldberg classifications from stage I to $V$.

Results: Retinopathy related to sickle cell anemia was seen in 62.3\% (38/61) of the eyes checked. Neovascularization was observed in one eye. The frequency of bilateral changes in angiography was high. Non-proliferative retinopathy was more common, especially vascular tortuosities (17/61), followed by arteriovenous anastomoses in the retinal periphery (15/61) and arterial occlusions (5/61). The mean age of retinopathy group was 14.4 years old, significantly lower than the mean age of non-retinopathy group, which was 17.4. The result was normal in $16.4 \%$ (10/61) of the eyes in the fundoscopy exam, while angiography showed alterations.

Conclusions: All the results pointed to the conclusion that the non-proliferative retinal vascular changes are frequent and precocious in patients with sickle cell anemia (SS genotype). Fluorescein angiography is more sensitive in the diagnosis of retinopathy when compared to indirect ophthalmoscopy.
\end{abstract}

Keywords: Anemia, sickle cell; Retina/physiopathology; Retinal diseases/diagnosis; Fluorescein angiography/methods; Ophthalmoscopy

\section{INTRODUÇÃO}

A anemia falciforme é a doença hereditária mais prevalente no

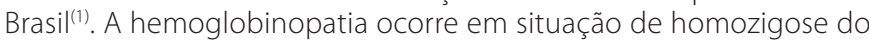
gene responsável pela produção de uma hemoglobina mutante, a hemoglobina $\mathrm{S}$, que em seu estado desoxigenado pode sofrer polimerização e originar hemácias falcizadas, responsáveis por anemia hemolítica crônica e fenômenos vasoclusivos ${ }^{(1-6)}$.

Embora haja outros genótipos para a doença falciforme, acredita-se que SC, S $\beta^{0}$ talassemia, em menor grau, o SS (este o responsável pelo fenótipo da anemia falciforme) resultem em maior risco para complicações oculares ${ }^{(2,4,5)}$.

As manifestações oculares da anemia falciforme compreendem alterações orbitárias, conjuntivais, uveais, papilares e, principalmente, retinianas ${ }^{(7-10)}$. A retina periférica e a mácula parecem ser os locais mais suscetíveis à oclusão vascular, onde as lesões são mais evidentes e destrutivas, podendo ocasionar cegueira ${ }^{(11-13)}$.

O presente estudo objetiva descrever e classificar alterações retinianas encontradas em portadores de anemia falciforme, bem
Submetido para publicação: 24 de maio de 2012

Aceito para publicação: 17 de agosto de 2012

Trabalho realizado na Universidade Federal de Sergipe em parceria com o Instituto de Olhos Dr. Cristiano Mendonça, Aracajú - Sergipe.

Médico, Departamento de Oftalmologia, Fundação Altino Ventura, Recife (PE), Brasil.

${ }^{2}$ Acadêmico de Medicina, Universidade Federal de Sergipe, Aracaju (SE), Brasil.

${ }^{3}$ Médico, Universidade Federal de Sergipe, Aracaju (SE), Brasil.

${ }^{4}$ Médico, Instituto de Olhos Cristiano Mendonça, Aracaju (SE), Brasil.
Financiamento: Não houve financiamento para este trabalho.

Divulgação de potenciais conflitos de Interesse: A.M.Santos, Nenhum; G.B.A.Faro, Nenhum; M.V.M.Amaral, Nenhum; C.Q.Mendonça, Nenhum; B.C.Leal, Nenhum; R.Cipolotti, Nenhum. Endereço de correspondência: Rosana Cipolotti, Av. Beira Mar, 2016, Apto. 402, Aracaju (SE) 49025-040, Brasil - E-mail: rosanaci@yahoo.com

Comitê de ética responsável pela aprovação: Comitê de Ética em Pesquisa Envolvendo Seres Humanos da Universidade Federal de Sergipe (CEP-UFS) №.CAAE:0140.0.107.000-07 
como comparar os métodos diagnósticos (mapeamento de retina e angiofluoresceinografia).

\section{MÉTODOS}

Foram avaliados neste estudo transversal (com dados obtidos prospectivamente), pacientes portadores de anemia falciforme, confirmada laboratorialmente por eletroforese de hemoglobina em $\mathrm{pH}$ alcalino, com idade igual ou superior a sete anos, em acompanhamento regular em ambulatório de Hematologia Pediátrica de Hospital Universitário da região nordeste do Brasil. Os pacientes foram consecutivamente encaminhados para avaliação oftalmológica, após assinatura de Termo de Consentimento Livre e Esclarecido pelo paciente ou seu responsável legal.

O exame oftalmológico consistiu em medida da acuidade visual corrigida através dos optotipos de Snellen, tonometria de aplanação de Goldmann, biomicroscopia com lâmpada de fenda, oftalmoscopia binocular indireta, retinografia e angiografia com injeção de fluoresceína sódica a 20\%, sendo esse três últimos após a midríase medicamentosa com tropicamida a 1\%. Todos os pacientes foram submetidos a todos os procedimentos citados.

Os achados fundoscópicos foram agrupados em: (1) sem alterações; (2) retinopatia não-proliferativa: tortuosidade venosa, hemorragias "salmon patch" (coloração alaranjada advinda da degradação da hemoglobina) e "blacksunburst" (lesão hiperpigmentada) $)^{(2,4,6,13)}$, pontos iridescentes, anastomoses arteriovenosas ${ }^{(5,14)}$, alterações na mácula e no nervo óptico (pontos escuros, pequenos evermelhos) ${ }^{(15,16)}$; (3) retinopatia proliferativa, caracterizada por neovascularização periférica denominada "seafun" $(2,5,6,13)$.

Os achados da angiofluoresceinografia foram descritos através da classificação de Goldberg(14) em cinco estágios, sendo os dois primeiros pré-proliferativos e os três restantes proliferativos ${ }^{(4,6,13,15)}$ : (I) oclusões arteriolares na periferia da retina; (II) anastomoses arteriovenosas na periferia da retina; (III) proliferações fibrosas e neovasculares; (IV) hemorragia vítrea; (V) descolamento da retina.

As variáveis categóricas foram apresentadas como porcentagens e a comparação entre os grupos, realizada por meio do teste de Fisher. As variáveis contínuas foram apresentadas por meio de medidas de tendência central e comparadas pelo teste T ou Mann Whitney, considerando-se significativos os valores de $p<0,05$. Os dados foram tabulados sem planilha Excel e analisados por meio do software Epilnfo versão 3.5.1.

\section{RESULTADOS}

Foram estudados 61 olhos de 31 pacientes (em um dos pacientes, um olho não pode ser avaliado por apresentar catarata traumática) portadores de anemia falciforme, dos quais 19 (61,3\%) eram do sexo masculino. A média de idade da amostra foi de 15,4 \pm 5,3 anos (14,4 anos nos pacientes com retinopatia x 17,4 anos nos sem alterações, $p=0,04)$. A hemoglobina basal média dos pacientes do estudo foi de $8,1 \pm 1,2 \mathrm{mg} / \mathrm{dL}(8,3 \mathrm{mg} / \mathrm{dL}$ no grupo dos com retinopatia e $7,8 \mathrm{mg} / \mathrm{dL}$ no sem alterações, $\mathrm{p}=0,19)$. Considerando-se em conjunto os resultados do mapeamento de retina e da angiofluoresceinografia, observou-se 38 olhos (19 pacientes) com retinopatia falciforme $(62,3 \%)$ e 23 (12 pacientes) sem alterações $(p<0,001)$, conforme consta na tabela1.

Ao mapeamento de retina encontraram-se 33 olhos normais e 28 com alterações do tipo não proliferativas. Na angiofluoresceinografia foram vistos 23 olhos normais, 17 com tortuosidades vasculares e 5 com oclusões arteriolares na retina periférica (estágio I de Goldberg), $15 \mathrm{com}$ anastomoses arteriovenosas na periferia da retina (estágio II) e 1 com proliferações neovasculares (estágio III). Ao mapeamento de retina, 14 pacientes apresentaram retinopatia bilateral relacionada à doença falciforme.
Dezoito pacientes possuíam alterações bilaterais na angiofluoresceinografia. Em 1 olho foi observada neovascularização. Nesse caso específico e outros 9 casos considerados normais pelo mapeamento de retina foram diagnosticados por meio da angiofluoresceinografia, diferença estatisticamente significante $(p<0,001)$, mostrando maior sensibilidade deste método em detrimento àquele, conforme consta na tabela 2.

\section{DISCUSSÃO}

Estudos anteriores apontaram frequências elevadas de retinopatia em portadores de hemoglobinopatia SC e S $\beta^{0}$ talassemia ${ }^{(2,4,5)}$. Entretanto, por ser o genótipo SS o mais frequente no Brasil e ainda pouco estudado, foi esse o grupo avaliado neste estudo.

Considerando-se em conjunto os resultados do mapeamento de retina e da angiofluoresceinografia observou-se retinopatia relacionada à anemia falciforme em $62,3 \%$ dos olhos estudados, achado compatível com a literatura, cujos dados apontam que mais de $50 \%$ dos pacientes apresentam pelo menos tortuosidade vascular ${ }^{(15,17)}$ Os achados mais frequentes foram de retinopatia não proliferativa, especialmente tortuosidades vasculares, seguidas por anastomoses arteriovenosas na periferia da retina e oclusões arteriolares, compatíveis com outros estudos ${ }^{(5,8,13)}$.

Observou-se, na amostra estudada, que a média de idade do grupo de portadores de retinopatia foi significantemente inferior à do grupo sem retinopatia. Esses achados são semelhantes aos da literatura ${ }^{(14)}$ e reafirmam a tendência de início precoce dessas alterações em pacientes susceptíveis. Observa-se, no entanto, que características como gênero ou hemoglobina média não estão associadas à presença de retinopatia.

Comparando-se os resultados do mapeamento de retina com os obtidos pela angiofluoresceinografia, observou-se correspondência quando os resultados foram normais. Entretanto em 16,4\% dos ol hos obteve-se resultado normal no mapeamento de retina, enquanto que a angiofluoresceinografia mostrou-se alterada. Portanto, a sensibilidade do mapeamento de retina foi inferior à da angiofluoresceinografia para a detecção de alterações retinianas ${ }^{(5)}$.

Tabela 1. Características clínicas dos pacientes portadores de anemia falciforme conforme presença de alterações retinianas ao mapeamento de retina e à angiofluoresceinografia

\begin{tabular}{lcccc}
\hline & $\begin{array}{c}\text { Amostra } \\
\text { total }\end{array}$ & $\begin{array}{c}\text { Grupo com } \\
\text { alterações }\end{array}$ & $\begin{array}{c}\text { Grupo sem } \\
\text { alterações }\end{array}$ & p \\
\hline No de olhos & 61 & 38 & 23 & $<0,001^{*}$ \\
Idade média (anos) & 15,4 & 14,4 & 17,4 & $0,040^{*}$ \\
Gênero masculino & 19 & 11 & 8 & $0,540^{+}$ \\
$\begin{array}{l}\text { Hemoglobina } \\
\text { média (mg/dL) }\end{array}$ & 8,1 & 8,3 & 7,8 & $0,190^{*}$ \\
\hline
\end{tabular}

${ }^{*}=$ teste Mann Whitney; ${ }^{\dagger}=$ teste Exato de Fisher.

Tabela 2. Correlação entre os achados ao mapeamento de retina e à angiofluoresceinografia em pacientes portadores de anemia falciforme

\begin{tabular}{lcccc}
\hline & \multicolumn{4}{c}{ Mapeamento de retina } \\
\cline { 2 - 5 } Angiofluoresceinografia & Normal & Alterada & Total & $\mathbf{p}$ \\
\hline Normal & 23 & 0 & 23 & \\
Alterada & 10 & 28 & 38 & $<0,001^{*}$ \\
Total & 33 & 28 & 61 & \\
\hline
\end{tabular}

*= teste "t" para amostras pareadas 
Observou-se frequência elevada de alterações bilaterais à angiofluoresceinografia neste estudo. Downes et al. observaram a possibilidade de reversão para unilateral em cerca de 30\% dos casos, o que indica que esses pacientes devem ser acompanhados clinicamente, mesmo não havendo indicação de intervenção terapêutica no momento(12).

Considerando-se que o paciente se mantém assintomático até estágios avançados, a identificação precoce de retinopatia proliferativa permite intervenções terapêuticas curativas. Não parece haver consenso com relação à idade apropriada para início do tratamento da retinopatia proliferativa no paciente portador de anemia falciforme, uma vez que existem relatos controversos sobre a eventual resolução espontânea do quadro(11), que, no entanto, não foi observada por outros autores ${ }^{(9)}$.

O presente estudo apresenta como limitações seu delineamento transversal e o reduzido tamanho da amostra estudada. Estudos longitudinais são necessários para ratificar achados aqui presentes e estabelecer fatores de risco para a retinopatia em pacientes portadores de anemia falciforme.

\section{CONCLUSÃO}

Com base nos casos estudados, que as alterações retinianas do tipo retinopatia não proliferativa, especialmente as tortuosidades vasculares, anastomoses arteriovenosas na periferia da retina e oclusões arteriolares são frequentes e precoces nos portadores de anemia falciforme, sendo a angiofluoresceinografia mais sensível no diagnóstico quando comparada ao mapeamento de retina. Os achados indicam a necessidade de acompanhamento oftalmológico, cuja periodicidade deve ser determinada pela extensão e gravidade das alterações encontradas.

\section{REFERÊNCIAS}

1. Paiva e Silva RB, Ramalho AS, Cassorla RM. A anemia falciforme como problema de Saúde Pública no Brasil. Rev Saúde Pública. 1993;27(1):54-8.
2. Freitas LG, Isaac DL, Tannure WT, Lima EV, Abud MB, Tavares RS, et al. Alterações retinianas apresentadas em pacientes portadores de hemoglobinopatia falciforme atendidos em um Serviço Universitário de Oftalmologia. Arq Bras Oftalmol. 2011; 74(5):335-7.

3. Bunn HF. Pathogenesis and treatment of sickle cell disease. N Engl J Med. 1997; 337(11):762-9.

4. Vilela RQ, Bandeira DM, Silva MA. Ocular complications in sickle cell disease. Rev Bras Hematol Hemoter. 2007;29(3):285-7.

5. Gill HS, Lam WC. A screening strategy for the detection of sickle cell retinopathy in pediatric patients. Can J Ophthalmol. 2008;43(2):188-91.

6. Bonanomi MT. Alterações oculares na doença falciforme. In: Agência Nacional de Vigilância Sanitária. Manual de diagnóstico e tratamento de doenças falciformes [Internet]. Brasília: ANVISA; 2002. p.97-105. [citado 2004 Jul 27]. Disponível em: http://bvsms.saude.gov.br/bvs/publicacoes/anvisa/diagnostico.pdf

7. Osafo-Kwaako A, Kimani K, Ilako D, Akafo S, Ekem I, Rodrigues O, et al. Ocular manifestations of sickle cell disease at the Korle-bu Hospital Accra, Ghana. Eur J Ophthalmol. 2010;21(4):484-9.

8. Morel C. [Retinal involvmente in hemoglobinopathy] J Fr Ophtalmol. 2001; 24(9).967-92. French.

9. Bisol T, Fior O, Esteves JF, Friderich JR. Sickle cell hemoglobinopathy genotypes and retinal manifestations in patients of a university hospital. Arq Bras Oftalmol. 2000;63(4):273-6.

10. Fadugbagbe AO, Gurgel RQ, Mendonça CQ, Cipolotti R, Santos AM, Cuevas LE. Ocular manifestations of sickle cell disease. Ann Trop Paediatr. 2010;30(1):19-26.

11. Elagouz M, Jyothi S, Gupta B, Sivaprasad S. Sickle cell disease and the eye: old and new concepts. Surv Ophthalmol. 2010;55(4):359-77.

12. Downes SM, Hambleton IR, Chuang EL, Lois N, Serjeant GR, Bird AC. Incidence and natural history of proliferative sickle cell retinopathy: observations from a cohort study. Ophthalmology. 2005;112(11):1869-75.

13. Bonanomi MT, Cunha SL, Araújo JT. Funduscopic alterations in SS and SC hemoglobinopathies - Study of a Brazilian population. Ophthalmologica.1988;197(1): 26-33.

14. Goldberg MF. Classification and pathogenesis of proliferative sickle cell retinopathy. Am J Ophthalmol. 1971;85(4):426-37.

15. Garcia CA, Fernandes MZ, Uchôa UB, Cavalcante BM, Uchôa RA. Achados fundoscópico sem crianças portadoras de anemia falciforme no estado do Rio Grande do Norte. Arq Bras Oftalmol. 2002;65(5):615-8.

16. Cook WC. A case of sickle cell anemia with associated subarachnoid hemorrhage. J Med. 1930;11:54117.

17. Bonanomi MT, Oliveira AA, Suzuki H. Hemoglobinopatias. In: AbujamraS, Ávila M, Barsante C. Retina e vítreo. São Paulo: Roca; 2000. p.592-601. 